\title{
Enquête sur la construction des Lumières, dir. F. SALAÜN et J.-P. SCHANDELER
}

Olga Penke

\section{(QpenEdition \\ Journals}

Édition électronique

URL : https://journals.openedition.org/studifrancesi/22646

DOI : 10.4000/studifrancesi.22646

ISSN : 2427-5856

\section{Éditeur}

Rosenberg \& Sellier

\section{Édition imprimée}

Date de publication : 1 avril 2020

Pagination : 173-174

ISSN : 0039-2944

Référence électronique

Olga Penke, "Enquête sur la construction des Lumières, dir. F. salaün et J.-P. schandeler 》, Studi Francesi [En ligne], 190 (LXIV | I) | 2020, mis en ligne le 01 mai 2020, consulté le 03 août 2021. URL : http:// journals.openedition.org/studifrancesi/22646 ; DOI : https://doi.org/10.4000/studifrancesi.22646

Ce document a été généré automatiquement le 3 août 2021.

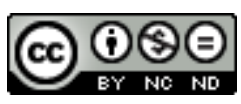

Studi Francesi è distribuita con Licenza Creative Commons Attribuzione - Non commerciale - Non opere derivate 4.0 Internazionale. 


\title{
Enquête sur la construction des Lumières, dir. F. SALAÜN et J.-P. SCHANDELER
}

\author{
Olga Penke
}

\section{RÉFÉRENCE}

Enquête sur la construction des Lumières, dir. F. SALAÜN et J.-P. SCHANDELER, Ferney-Voltaire, Centre International d'Études du XviII ${ }^{\mathrm{e}}$ siècle, 2018, 254 pp.

1 Les Lumières, terme de l'histoire littéraire, de la philosophie et de l'histoire des sciences fort débattu de nos jours est employé trop vaguement, en particulier quand on parle de son esprit, héritage ou de ses messages. La question de «globalisation» de cette catégorie et un essai de clarification de ses contenus sont mis au centre de ce livre, issu d'un séminaire de recherches. Les deux directeurs du volume ont réparti les études de dix-huit intervenants autour de trois champs d'enquête: les antécédents, les autodéfinitions, les contenus et les appréciations (I. «Ouverture» et II. «Généalogie»), les interprétations dans des contextes géopolitiques divers (III. «Géographie»), les appropriations, les négations (IV. «Usage»). Quelques auteurs saillants (Rousseau, Diderot, Voltaire, Condorcet) et quelques œuvres majeures (l'Encyclopédie, l'Histoire des deux Indes) sont mis en relief par plusieurs intervenants.

Dans la première section du volume Franck SALAÜN (L'objet «Lumières»: problèmes et perspectives, pp. 9-24) présente une analyse lucide des questions les plus discutées concernant le syntagme Lumières, et cherche à découvrir le point de départ et les causes des débats qui se déroulent dans les lieux divers du monde d'aujourd'hui sur ses valeurs et sa chronologie. Il propose, en tant que solution provisoire, sa délimitation, pour «l'affirmation des principes du débat et de la liberté de pensées», ayant une certaine homogénéité dans les années 1750-1780. Jean-Pierre SCHANDELER dans La construction des Lumières par l'édition et la traduction (pp. 25-33) analyse pour sa part le 
cas de Condorcet pour montrer comment les $\mathrm{XIX}^{\mathrm{e}}$ et $\mathrm{xx}^{\mathrm{e}}$ siècles cherchent à réactualiser ses œuvres en France, et comment les traductions les réintègrent aux débats nationaux de leurs propres. Les études de la deuxième partie traitent des particularités et des écarts des pensées des philosophes français. Eszter kovács (Les stratégies défensives des Lumières. Autour de la "Défense de l'Esprit des lois", pp. 37-46) présente le cas singulier de Montesquieu qui s'abstient généralement des débats, mais qui, après les critiques adressées à L'Esprit des lois, consacre à la défense de son livre, en 1750, une œuvre entière, dans laquelle il souligne l'idéal de la critique juste et du ton modéré dans les discussions. Hans-Jürgen LÜSEBRINK ("Lumières» et «ténèbres» dans le discours philosophique et historiographique du XVIII 'siècle: l'exemple de l'“Histoire des deux Indes", pp. 47-60) analyse finement la présence marquée et les significations variées du couple sémantique «Lumières» et "ténèbres» dans l'ouvrage historique et politique ayant une influence jusqu'à nos jours, conçu et rédigé par une équipe d'auteurs parmi lesquels se distinguent Raynal, Diderot, Naigeon, Deleyre et Pechmeja. Selon Muriel вRот (Le rôle des administrateurs coloniaux dans l'écriture de l"'Histoire des deux Indes", pp. 61-78) les contradictions de cet ouvrage découlent d'une dispute historiographique fondamentale des auteurs sur la manière convenable d'écrire l'histoire coloniale. L'historien «calculateur», s'intéressant aux faits quantitatifs afin de réformer l'administration des colonies, acceptant que l'esclavage est indispensable à leur exploitation, est juxtaposé à l'historien philosophe, «éloquent», s'opposant radicalement aux principes politiques et économiques de l'esclavage, et mettant en question la justesse de la colonisation. Les oppositions extrêmes peuvent également se présenter à l'intérieur de l'œuvre d'un auteur. Giovanni PAOLETTI (Les Lumières et l'art de tromper: le cas de Condorcet, pp. 79-97) analyse la réflexion de Condorcet avant et pendant la Révolution concernant la question des altérations de la vérité et de la nécessité de la tromperie. Il constate le rapport complexe entre le phénomène d'éclairer et de dire la vérité au peuple, ainsi que le renversement de l'opinion du philosophe à la fin de sa vie, quand il considère la tromperie comme moyen de résistance à l'oppression. La conception fondamentalement diverse de l'idéal des Lumières peut être égalent remarquée dans la littérature nationale entre les auteurs différents, comme le démontre Petr HORÂK (L'ambiguïté des Lumières selon Jan Patočka, pp. 99-102), par les exemples de la littérature du XviII ${ }^{\mathrm{e}}$ siècle allemand.

3 La troisième section concentre l'intérêt sur les changements internationaux, européens et américains, ayant un rôle dans le mouvement des Lumières. Gilles DENIS (Sociétés d'agriculture et diffusion des Lumières, pp. 105-118) réfléchit sur le fonctionnement efficace des sociétés d'agriculture anglaises, écossaises, irlandaises, américaines, dans la diffusion d'une culture commune agricole, et prouve que leur activité a largement contribué à ce que l'agriculture devienne non seulement une pratique (amélioration des terres incultes, développement des instruments, des manufactures, du commerce agricoles), mais également une science autonome. Marc MARTI (Les Lumières espagnoles et la quête du bonheur (1746-1814), pp. 119-131) démontre, par l'examen des champs sémantiques des discours politiques et économiques espagnols, que le bonheur constitue leur idée centrale à partir $\mathrm{du} \mathrm{xvI}^{\mathrm{e}}$ siècle, tandis que dès la seconde moitié du $\mathrm{xVIII}^{\mathrm{e}}$ siècle une tension est perceptible au niveau du vocabulaire se rapportant au bonheur, qu'on peut mettre en rapport avec la transformation de l'idéal de la conception monarchique en celui du système constitutionnel; le premier réduisant l'individu au passif, le second soulignant son appartenance à une collectivité et sa 
participation active. À travers une analyse centrée sur le lexique des articles du périodique "El Censor", Sylvie IMPARATO-PRIEUR (Une autodéfinition des Lumières espagnoles: "El Censor" (1781-1787), pp. 133-143) éclaire l'intention des journalistes d'inscrire l'Espagne dans le mouvement européen des Lumières en tant que nation sur la courbe ascendante du progrès. Luiz Carlos VILLALTA (Les appropriations des Lumières par les conspirateurs du Minas Gerais et de Bahia: textes, auteurs et débats au Brésil à la fin du XVIII ${ }^{\mathrm{e}}$ siècle, pp. 145-155) met en relief les différences fondamentales entre deux conspirations contre le régime portugais au Brésil, se déroulant avec quelques années d'écart, dont les participants cherchent à appliquer certains textes des Lumières françaises au cours de leur combat. La première, dirigée par des gens ayant une formation spécifique (clercs, avocats, militaires, etc.) qui se révoltent afin d'établir des réformes, se sert de l'argumentation de l'Histoire des deux Indes, tandis que la seconde, organisée par les mécontents originaires du pays (noirs, mulâtres, esclaves), puisent leurs idées beaucoup plus radicales dans les textes nés sous ou après la Révolution française. Ériona TARTARI KËRTUSHA (La Renaissance albanaise entre le Romantisme et les Lumières, pp. 157-164) traite de la difficulté de parler de l'influence des Lumières en Albanie, ce terme, tout comme celui de renaissance, romantisme, symbolisme, étant appliqué dans un sens divergeant de l'utilisation commune, ce qu'elle met en rapport avec la situation historique particulière du pays qui devient indépendant au $\mathrm{xx}^{\mathrm{e}}$ siècle et où même l'alphabet latin est institutionnalisé seulement en 1908.

Dans la dernière partie du volume sont regroupées les études qui mettent en relief l'utilisation abusive, voire l'appropriation du syntagme Lumières. Christian GILAIN (Les Lumières dans l'historiographie des mathématiques, pp. 167-179) avertit que la notion de «mathématique des Lumières» peut mener aux simplifications extrêmes et démontre que la réflexion de D'Alembert, de Condorcet, de Bougainville, d'Euler ou de Mlle Agnesi est foncièrement différente, tandis que chacun s'éloigne de la conception étroitement utilitaire. La contribution d'Isabel DRUMOND BRAGA (Musées d'histoire naturelle et jardins botaniques au Portugal: nature, divertissement et éducation (XVIII et XIX ${ }^{\mathrm{e}}$ siècles), pp. 181-191) établit la situation particulière de l'histoire de l'étude de la nature au Portugal qui commence par les voyageurs dès le $\mathrm{XVI}^{\mathrm{e}}$ siècle et aboutit à des collections précieuses (cabinets de curiosités), détruites au cours du tremblement de la terre de 1755. Cet intérêt, soutenu aussi bien par les Académies que par la politique, se tournera dès la fin du XVIII ${ }^{e}$ siècle vers des objectifs utilitaires, cherchant à développer l'enseignement et l'économie. Alla polosina (Les traductions de Rousseau en Russie. Quelques interprétations de son ceuvre, pp. 193-199) démontre par de nombreux exemples la popularité exceptionnelle de Rousseau en Russie parmi les philosophes et écrivains russes du XIX siècle et fait un tour d'horizon de la traduction de ses œuvres depuis le $\mathrm{xVIII}^{\mathrm{e}}$ siècle à nos jours. Pascale PELLERIN (Les Lumières: un enjeu idéologique pendant la guerre d'Algérie (1954-1962), pp. 201-217) analyse un grand corpus journalistique, publié en français par les indépendantistes algériens et par les auteurs franco-français de gauche et de droite, afin de prouver que les textes de Voltaire et de Diderot sont cités des deux côtés dans un sens très hétérogène au cours de la lutte idéologique pour et contre l'indépendance de cette colonie française de statut exceptionnel. Marie-Noëlle CICCIA (Le tremblement de terre de Lisbonne et les Lumières au Portugal selon Miguel Real, pp. 219-230) cherche à montrer, à travers l'analyse des œuvres d'un philosophe portugais de nos jours, que les réformes de Pombal ont universalisé et altéré le caractère de la culture traditionnelle de son pays, perte irréparable. L'image de l'Afrique, présentée dans les articles de 
l'Encyclopédie analysée par David DIoP avec l'appui d'une large littérature critique, lui permet de mettre en question l'humanisme universel des Lumières (Les Lumières et l'Afrique: usages de l'humanisme, pp. 231-240). Il atteste que les philosophes ne reconnaissant pas le caractère inhumain de l'esclavage ont fourni un alibi aux colonisations.

5 Ce recueil bien constitué, qui réunit les idées coexistantes dans les recherches internationales concernant les Lumières, procure de nombreux résultats nouveaux, permet de mieux comprendre comment la généralisation extrême et la rencontre des cultures divergentes ont entraîné la mise en question du terme Lumières dans le discours d'aujourd'hui et peut finalement contribuer à sa redéfinition souhaitée. 